УДК 519.644

\title{
Optimal Formulas of Numerical Integration with Derivatives in Sobolev Space
}

\author{
Kholmat M. Shadimetov \\ Farhod A. Nuraliev* \\ Institute of Mathematics \\ Uzbekistan Academy of Sciences
}

Mirzo Ulugbek, 81, Tashkent, 100041

Uzbekistan

Received 05.10.2017, received in revised form 27.03.2018, accepted 09.07.2018

The problem of construction of optimal quadrature formulas in the sense of Sard in the space $L_{2}^{(m)}(0,1)$ is considered in the paper. The quadrature sum consists of values of the integrand at internal nodes and values of the first, third and fifth derivatives of the integrand at the end points of the integration interval. The coefficients of optimal quadrature formulas are found and the norm of the optimal error functional is calculated for arbitrary natural number $N$ and for any $m \geqslant 6$ using Sobolev method. It is based on discrete analogue of the differential operator $d^{2 m} / d x^{2 m}$. In particular, for $m=6,7$ optimality of the classical Euler-Maclaurin quadrature formula is obtained. Starting from $m=8$ new optimal quadrature formulas are obtained.

Keywords: optimal quadrature formula, error functional, extremal function, Sobolev space, optimal coefficients.

DOI: 10.17516/1997-1397-2018-11-6-764-775.

\section{Introduction. Statement of the problem}

We consider the following general quadrature formula

$$
\int_{0}^{1} \varphi(x) d x \cong \sum_{\beta=0}^{N} C[\beta] \varphi(h \beta)+\sum_{n=1}^{3} A_{n}\left(\varphi^{(2 n-1)}(0)-\varphi^{(2 n-1)}(1)\right)
$$

with the error functional

$$
\ell(x)=\varepsilon_{[0,1]}(x)-\sum_{\beta=0}^{N} C[\beta] \delta(x-h \beta)+\sum_{n=1}^{3} A_{n}\left(\delta^{(2 n-1)}(x)-\delta^{(2 n-1)}(x-1)\right)
$$

in the space $L_{2}^{(m)}(0,1)$ for $m \geqslant 6$. Here $C[\beta], \beta=\overline{0, N}$ and $A_{n}, n=1,2,3$ are the coefficients of formula (1.1), $h=\frac{1}{N}, N$ is a natural number, $\varepsilon_{[0,1]}(x)$ is the characteristic function on the interval $[0,1], \delta(x)$ is the Dirac delta-function and $\varphi$ is an element of the Sobolev space

$$
L_{2}^{(m)}(0,1):=\left\{\varphi:[0,1] \rightarrow \mathbb{R} \mid \varphi^{(m-1)} \text { is abs. cont. and } \varphi^{(m)} \in L_{2}(0,1)\right\}
$$

equipped with the norm

$$
\left\|\varphi \mid L_{2}^{(m)}(0,1)\right\|=\left\{\int_{0}^{1}\left(\varphi^{(m)}(x)\right)^{2} d x\right\}^{1 / 2}
$$

*nuraliyevf@mail.ru

(c) Siberian Federal University. All rights reserved 
and $\int_{0}^{1}\left(\varphi^{(m)}(x)\right)^{2} d x<\infty$

Equality (3) is a semi-norm and $\|\varphi\|=0$ if and only if $\varphi(x)=P_{m-1}(x)$ where $P_{m-1}(x)$ is a polynomial of degree $m-1$.

The difference

$$
(\ell, \varphi)=\int_{0}^{1} \varphi(x) d x-\sum_{\beta=0}^{N} C[\beta] \varphi(h \beta)-\sum_{n=1}^{3} A_{n}\left(\varphi^{(2 n-1)}(0)-\varphi^{(2 n-1)}(1)\right)
$$

is called the error of quadrature formula (1.1).

By the Cauchy-Schwarz inequality

$$
|(\ell, \varphi)| \leqslant\left\|\varphi\left|L_{2}^{(m)}(0,1)\|\cdot\| \ell\right| L_{2}^{(m)^{*}}(0,1)\right\|
$$

error (1.4) of formula (1.1) is estimated by the norm of error functional (1.2) in the conjugate space $L_{2}^{(m)^{*}}(0,1)$, i.e, by

$$
\left\|\ell\left|L_{2}^{(m)^{*}}(0,1) \|=\sup _{\left\|\varphi \mid L_{2}^{(m)}(0,1)\right\|=1}\right|(\ell, \varphi) \mid\right.
$$

Thus estimation of error (1.4) of quadrature formula (1.1) on functions in the space $L_{2}^{(m)}(0,1)$ is reduced to finding the norm of the error functional $\ell$ in the conjugate space $L_{2}^{(m)^{*}}(0,1)$.

Obviously the norm of the error functional $\ell$ depends on coefficients and nodes of quadrature formula (1.1). The problem of finding the minimum of the norm of the error functional $\ell$ that depends on coefficients and nodes is called Nikol'skii problem. The resulting formula is called optimal quadrature formula in the sense of Nikol'skii. This problem was first considered by S. M. Nikol'skii [11], and by many authors (see e.g. [1-4,12,29] and references therein). Minimization of the norm of the error functional $\ell$ in terms of coefficients when the nodes are fixed is called the Sard problem. The resulting formula is called the optimal quadrature formula in the sense of Sard. This problem was first investigated by A. Sard [13].

The results of this paper are related to the Sard problem. So here we discuss some of the previous results on optimal quadrature formulas in the sense of Sard which are closely related to our results.

There are several methods of construction of optimal quadrature formulas in the sense of Sard such as spline method, $\varphi$-function method (see e.g. $[1,15])$ and the Sobolev method which is based on construction of discrete analogue of a linear differential operator (see e.g. [25, 26]). The Sard problem was investigated in various spaces by many authors (see, for example, $[1,3,7$ 10,14-16, 18, 20,21, 24-28] and references therein).

The main aim of this paper is to construct optimal quadrature formulas (1.1) in the sense of Sard in the space $L_{2}^{(m)}(0,1)$.

We use the Sobolev method $[25,26]$ which is based on the discrete analogue of the differential operator $d^{2 m} / d x^{2 m}$.

In order to define error functional $(1.4)$ in the space $L_{2}^{(m)}(0,1)$ it should satisfy the following conditions (see [24])

$$
\left(\ell, x^{\alpha}\right)=0, \quad \alpha=0,1,2, \ldots, m-1 .
$$

Hence it is clear that for existence of quadrature formulas (1.1) the condition $N \geqslant m-4$ should be satisfied.

As it was noted above the error of formula (1.1) is estimated by the norm $\|\ell\|$ of error functional (1.2). Furthermore the norm of error functional (2) depends on coefficients $C[\beta], A_{n}$. 
We minimize the norm of error functional (2) in terms of coefficients $C[\beta]$ and $A_{n}$, i.e., we find

$$
\left\|\stackrel{\ell}{ }\left|L_{2}^{(m) *}\left\|=\inf _{C[\beta], A_{n}}\right\| \ell\right| L_{2}^{(m) *}\right\| .
$$

Coefficients $C[\beta]$ and $A_{n}$ which satisfy (1.6) are called the optimal coefficients and they are denoted by $\stackrel{C}{C}[\beta]$ and $\AA_{n}$. The corresponding quadrature formula is called the optimal quadrature formula in the sense of Sard. For the sake of convenience the optimal coefficients $\dot{C}[\beta]$ and $\dot{A}_{n}$ will be denoted as $C[\beta]$ and $A_{n}$.

Thus to construct optimal quadrature formulas (1.1) in the sense of Sard we need to solve consequently the following problems.

Problem 1. Find the norm of error functional (1.2) of quadrature formula (1.1) in the space $L_{2}^{(m) *}(0,1)$.

Problem 2. Find coefficients $C[\beta]$ and $A_{n}$ which satisfy relation (1.6).

It should be noted that many mathematicians have contributed to the development of the theory of cubature and quadrature formulas among them S. L. Sobolev, I. P. Mysovskikh, V. I. Lebedev, M. D. Ramazanov, G. N. Salikhov, V. I. Polovinkin, M. V. Noskov, V. L. Vaskevich and others.

The paper is organized as follows: in Section 2 we give representation of the norm of error functional (1.2) and we obtain the system of linear equations for the coefficients of the optimal quadrature formulas in the space $L_{2}^{(m)}(0,1)$. Moreover, the existence and uniqueness of the solution of this system are discussed; in Section 3, using the discrete analogue of the operator $d^{2 m} / d x^{2 m}$, explicit formulas for coefficients of optimal quadrature formulas (1.1) are found, and the norm of error functional (1.2) is calculated.

\section{The system of equations for optimal coefficients}

To solve Problem 1 we get the following expression for the norm of error functional (1.2) [23]

$$
\begin{gathered}
\|\ell\|^{2}=(-1)^{m}\left[\sum_{\beta=0}^{N} \sum_{\gamma=0}^{N} C[\beta] C[\gamma] \frac{|h \beta-h \gamma|^{2 m-1}}{2(2 m-1) !}-2 \sum_{\beta=0}^{N} C[\beta] \int_{0}^{1} \frac{|x-h \beta|^{2 m-1}}{2(2 m-1) !} d x+\right. \\
\quad+2 \sum_{n=1}^{3} A_{n} \int_{0}^{1} \frac{\left(x^{2 m-2 n}+(1-x)^{2 m-2 n}\right)}{2(2 m-2 n) !} d x- \\
-2 \sum_{n=1}^{3} A_{n} \sum_{\beta=0}^{N} C[\beta] \frac{\left((h \beta)^{2 m-2 n}+(1-h \beta)^{2 m-2 n}\right)}{2(2 m-2 n) !}+ \\
\left.\quad+\sum_{n=1}^{3} \sum_{k=1}^{3} \frac{A_{n} A_{k}}{(2 m-2 n-2 k+1) !}+\frac{1}{(2 m+1) !}\right] .
\end{gathered}
$$

Further, in order to solve Problem 2 we apply the Lagrange method, i.e., to find the minimum of $\|\ell\|^{2}$ under conditions (1.5) we consider the following function

$$
\Psi=\|\ell\|^{2}-2 \cdot(-1)^{m} \sum_{\alpha=0}^{m-1} \lambda_{\alpha}\left(\ell(x), x^{\alpha}\right),
$$

where $\lambda_{\alpha}$ are unknown multipliers. The function $\Psi$ is the multidimensional function with respect to coefficients $C[\beta], A_{n}$ and $\lambda_{\alpha}$. Setting partial derivatives of $\Psi$ with respect to coefficients 
$C[\beta], A_{n}$ to zero and taking into account conditions (1.5), we obtain the following system of linear equations

$$
\begin{aligned}
& \sum_{\gamma=0}^{N} C[\gamma] \frac{|h \beta-h \gamma|^{2 m-1}}{2(2 m-1) !}-\sum_{n=1}^{3} A_{n} \frac{\left((h \beta)^{2 m-2 n}+(1-h \beta)^{2 m-2 n}\right)}{2(2 m-2 n) !}+ \\
& +\sum_{\alpha=0}^{m-1} \lambda_{\alpha}(h \beta)^{\alpha}=f_{m}(h \beta), \quad \beta=\overline{0, N} \\
& \left.\sum_{\beta=0}^{N} C[\beta] \frac{\left((h \beta)^{2 m-2 k}+(1-h \beta)^{2 m-2 k}\right)}{2(2 m-2 k) !}-\sum_{n=1}^{3} \frac{A_{n}}{2(2 m-2 n-2 k+1) !}\right)+ \\
& +\sum_{\alpha=2 k}^{m-1} \alpha(\alpha-1) \ldots(\alpha-2 k+2) \cdot \lambda_{\alpha}=\frac{1}{(2 m-2 k+1) !}, \quad k=1,2,3, \\
& \sum_{\beta=0}^{N} C[\beta](h \beta)^{\alpha}=\frac{1}{\alpha+1}, \quad \alpha=0,1 \\
& \sum_{\beta=0}^{N} C[\beta](h \beta)^{\alpha}-\alpha A_{1}=\frac{1}{\alpha+1}, \quad \alpha=2,3, \\
& \sum_{\beta=0}^{N} C[\beta](h \beta)^{\alpha}-\alpha A_{1}-\alpha(\alpha-1)(\alpha-2) A_{2}=\frac{1}{\alpha+1}, \quad \alpha=4,5, \\
& \sum_{\beta=0}^{N} C[\beta](h \beta)^{\alpha}-\alpha A_{1}-\alpha(\alpha-1)(\alpha-2) A_{2}- \\
& -\alpha(\alpha-1)(\alpha-2)(\alpha-3)(\alpha-4) A_{3}=\frac{1}{\alpha+1}, \quad \alpha=\overline{6, m-1}
\end{aligned}
$$

where

$$
f_{m}(h \beta)=\int_{0}^{1} \frac{|x-h \beta|^{2 m-1}}{2(2 m-1) !} d x=\frac{(h \beta)^{2 m}}{(2 m) !}+\sum_{j=0}^{2 m-1} \frac{(-h \beta)^{2 m-1-j}}{2(2 m-1-j) ! \cdot(j+1) !} .
$$

System (2.2)-(2.7) is called the discrete system of Wiener-Hopf type for the optimal coefficients $[24,26]$. Coefficients $C[\beta], \beta=\overline{0, N}, A_{n}$ and $\lambda_{\alpha}, \alpha=\overline{0, m-1}$ are unknowns of system (12)-(17). System (2.2)-(2.7) has unique solution and this solution gives the minimum of $\|\ell\|^{2}$. Here we omit the proof of the existence and uniqueness of the solution of this system. The proof of the existence and uniqueness of the solution of this system coincides with the proof of the existence and uniqueness of the solution of discrete Wiener-Hopf type system for the optimal coefficients in the space $L_{2}^{(m)}(0,1)$ for quadrature formulas of the form $\int_{0}^{1} f(x) d x \cong \sum_{\beta=0}^{N} C[\beta] f[\beta]$ (see $\left.[24,26]\right)$.

It should be noted that the uniqueness of the optimal quadrature formulas in the Sard sense was also discussed [9].

\section{The coefficients and the norm of the error functional of the optimal quadrature formulas}

In this section we solve system (2.2)-(2.7) and find the explicit formulas for coefficients $C[\beta]$. We use functions of discrete variable and operations on them (see [24,26]). 
First we consider some statements that are used in the proofs of main results.

The following theorem is devoted to representation of the Euler-Frobenius polynomial.

Theorem 3.1 (Lemma 3 of [17]). Polynomial

$$
Q_{k}(x)=(x-1)^{k+1} \sum_{i=0}^{k+1} \frac{\Delta^{i} 0^{k+1}}{(x-1)^{i}}
$$

is the Euler-Frobenius polynomial of degree $k$, i.e., $Q_{k}(x)=E_{k}(x)$ where $\Delta^{i} 0^{k}=\sum_{l=1}^{i}(-1)^{i-l} C_{i}^{l} l^{k}$.

We need the discrete analogue $D_{m}(h \beta)$ of the operator $d^{2 m} / d x^{2 m}$ which satisfies the following equation

$$
h D_{m}(h \beta) * G_{m}(h \beta)=\delta(h \beta),
$$

where $G_{m}(h \beta)=\frac{|h \beta|^{2 m-1}}{2(2 m-1) !}, \delta(h \beta)$ is equal to 0 when $\beta \neq 0$, and it is equal to 1 when $\beta=0$, i.e., $\delta(h \beta)$ is the discrete delta-function.

It should be noted that the operator $D_{m}(h \beta)$ was firstly introduced and investigated by S. L. Sobolev [24].

The discrete analogue $D_{m}(h \beta)$ of the differential operator $d^{2 m} / d x^{2 m}$ which satisfies equation (3.1) was constructed and the following theorem was proved [19].

Theorem 3.2. The discrete analogue of the differential operator $d^{2 m} / d x^{2 m}$ has the form

$$
D_{m}(h \beta)=\frac{(2 m-1) !}{h^{2 m}} \begin{cases}\sum_{k=1}^{m-1} \frac{\left(1-q_{k}\right)^{2 m+1} q_{k}^{|\beta|}}{q_{k} E_{2 m-1}\left(q_{k}\right)} & \text { for } \quad|\beta| \geqslant 2, \\ 1+\sum_{k=1}^{m-1} \frac{\left(1-q_{k}\right)^{2 m+1}}{E_{2 m-1}\left(q_{k}\right)} & \text { for } \quad|\beta|=1, \\ -2^{2 m-1}+\sum_{k=1}^{m-1} \frac{\left(1-q_{k}\right)^{2 m+1}}{q_{k} E_{2 m-1}\left(q_{k}\right)} & \text { for } \quad \beta=0,\end{cases}
$$

where $E_{2 m-1}(q)$ is the Euler-Frobenius polynomial of degree $2 m-1, q_{k}$ are roots of the EulerFrobenius polynomial $E_{2 m-2}(q),\left|q_{k}\right|<1, h$ is a small positive parameter.

Several properties of function $D_{m}(h \beta)$ were proved [19]. Here we consider the following property of function $D_{m}(h \beta)$.

Theorem 3.3. Function $D_{m}(h \beta)$ and monomials $(h \beta)^{k}$ are related to each other as follows

$$
\begin{gathered}
\sum_{\beta=-\infty}^{\infty} D_{m}(h \beta)(h \beta)^{k}=\left\{\begin{array}{lll}
0 & \text { when } & 0 \leqslant k \leqslant 2 m-1, \\
(2 m) ! & \text { when } & k=2 m,
\end{array}\right. \\
\sum_{\beta=-\infty}^{\infty} D_{m}(h \beta)(h \beta)^{k}=\left\{\begin{array}{lll}
0 & \text { when } & 2 m+1 \leqslant k \leqslant 4 m-1, \\
\frac{h^{2 m}(4 m) ! B_{2 m}}{(2 m) !} & \text { when } & k=4 m .
\end{array}\right.
\end{gathered}
$$

Then taking into account (3.1) and using (2.2), Theorems 3.2, 3.3, we have the following theorem for optimal coefficients $C[\beta]$. 
Theorem 3.4. Coefficients $C[\beta], \beta=1,2, \ldots, N-1$ of the optimal quadrature formulas (1) in the space $L_{2}^{(m)}(0,1), m \geqslant 6$, have the following form

$$
C[\beta]=h\left(1+\sum_{k=1}^{m-1}\left(d_{k} q_{k}^{\beta}+p_{k} q_{k}^{N-\beta}\right)\right), \quad \beta=1,2, \ldots, N-1,
$$

where $d_{k}, p_{k}$ are unknowns, $q_{k}$ are given in Theorem 3.2.

Theorem 3.4 is proved similarly as Theorem 5.3 [23].

Furthermore we need the following lemmas for the proof of the main results.

Lemma 1. The following relation is taken place [23]

$$
\sum_{i=0}^{\alpha} \frac{d_{k} q_{k}+p_{k} q_{k}^{N+i}(-1)^{i+1}}{\left(q_{k}-1\right)^{i+1}} \Delta^{i} 0^{\alpha}=(-1)^{\alpha+1} \sum_{i=0}^{\alpha} \frac{d_{k} q_{k}^{i}+p_{k} q_{k}^{N+1}(-1)^{i+1}}{\left(1-q_{k}\right)^{i+1}} \Delta^{i} 0^{\alpha},
$$

here $\alpha$ and $N$ are natural numbers, $d_{k}$ and $p_{k}$ are constants, $\Delta^{i} 0^{\alpha}$ is given in Theorem 3.1, $q_{k}$ are given in Theorem 3.2.

Lemma 2. The following relations are valid

$$
\begin{aligned}
\sum_{j=1}^{m-1} \frac{(-1)^{j-1}}{(j-1) !} \sum_{i=1}^{2 m-2-j} \frac{B_{2 m-j-i} h^{2 m-j-i}}{i !(2 m-j-i) !} & =\sum_{j=2}^{m} \frac{B_{j} h^{j}}{j !} \sum_{i=0}^{m-2} \frac{(-1)^{i}}{i !(2 m-1-j-i) !}+ \\
& +\sum_{j=m+1}^{2 m-2} \frac{B_{j} h^{j}}{j !} \sum_{i=0}^{2 m-2-j} \frac{(-1)^{i}}{i !(2 m-1-j-i) !}
\end{aligned}
$$

and

$$
\begin{aligned}
\sum_{j=1}^{m-1} \frac{(-1)^{j-1}}{(j-1) !} \sum_{p=1}^{2 m-1-j} \frac{h^{p+1} Z_{p}}{p !(2 m-1-j-p) !} & =\sum_{j=2}^{m+1} \frac{h^{j} Z_{j-1}}{(j-1) !} \sum_{i=0}^{m-2} \frac{(-1)^{i}}{i !(2 m-1-j-i) !}+ \\
& +\sum_{j=m+2}^{2 m-1} \frac{h^{j} Z_{j-1}}{(j-1) !} \sum_{i=0}^{2 m-1-j} \frac{(-1)^{i}}{i !(2 m-1-j-i) !}
\end{aligned}
$$

where

$$
Z_{p}=\sum_{k=1}^{m-1} \sum_{i=0}^{p} \frac{d_{k} q_{k}^{N+i}+p_{k} q_{k}(-1)^{i+1}}{\left(1-q_{k}\right)^{i+1}} \Delta^{i} 0^{p}
$$

The proof of Lemma 2 is obtained with the use of expansion of the left hand sides of given above relations in powers of $h$.

Finally we give the main results of the paper.

The following theorem is valid for the coefficients of the optimal quadrature formulas (1.1).

Theorem 3.5. Among quadrature formulas (1.1) with error functional (1.2) in the space $L_{2}^{(m)}(0,1), m \geqslant 6$ there exists the unique optimal formula which coefficients

$$
\begin{aligned}
& \stackrel{\circ}{C}[0]=h\left(\frac{1}{2}+\sum_{k=1}^{m-1} d_{k} \frac{q_{k}^{N}-q_{k}}{1-q_{k}}\right), \\
& \stackrel{\circ}{C}[\beta]=h\left(1+\sum_{k=1}^{m-1} d_{k}\left(q_{k}^{\beta}+q_{k}^{N-\beta}\right)\right), \quad \beta=\overline{1, N-1},
\end{aligned}
$$




$$
\begin{aligned}
\stackrel{\circ}{C}[N] & =h\left(\frac{1}{2}+\sum_{k=1}^{m-1} d_{k} \frac{q_{k}^{N}-q_{k}}{1-q_{k}}\right) \\
\stackrel{A}{n}_{n} & =\frac{h^{2 n} B_{2 n}}{(2 n) !}-\frac{h^{2 n}}{(2 n-1) !} \sum_{k=1}^{m-1} \sum_{i=1}^{2 n-1} d_{k} \frac{q_{k}+q_{k}^{N+i}(-1)^{i+1}}{\left(q_{k}-1\right)^{i+1}} \Delta^{i} 0^{2 n-1}, n=1,2,3,
\end{aligned}
$$

where $d_{k}$ satisfy the following system of $m-1$ linear equations

$$
\begin{aligned}
& \sum_{k=1}^{m-1} \sum_{i=0}^{j} d_{k} \frac{q_{k}+q_{k}^{N+i}(-1)^{i+1}}{\left(q_{k}-1\right)^{i+1}} \Delta^{i} 0^{j}=\frac{B_{j+1}}{j+1}, \quad j=\overline{6, m-1} \\
& \sum_{k=1}^{m-1} \sum_{i=0}^{2 m-2 j} d_{k} \frac{q_{k}+q_{k}^{N+i}(-1)^{i+1}}{\left(q_{k}-1\right)^{i+1}} \Delta^{i} 0^{2 m-2 j}=0, \quad j=1,2,3 \\
& \sum_{k=1}^{m-1} \sum_{i=0}^{2 j} d_{k} \frac{q_{k}+q_{k}^{N+i}(-1)^{i+1}}{\left(q_{k}-1\right)^{i+1}} \Delta^{i} 0^{2 j}=0, \quad j=1,2 .
\end{aligned}
$$

Here $B_{\alpha}$ are Bernoulli numbers, $\Delta^{i} \gamma^{j}$ is the finite difference of $\gamma^{j}$ of order $i, \Delta^{i} 0^{j}$ is given in Theorem 3.1, $q_{k}$ are given in Theorem 3.2.

For square of the norm of error functional (1.2) of optimal quadrature formulas (1.1) we give the following result without proof. The proof is given in Theorem 5.5 [23].

Theorem 3.6. For square of the norm of error functional (1.2) of the optimal quadrature formula (1.1) in the space $L_{2}^{(m)}(0,1), m \geqslant 6$ the following relation holds

$$
\left\|\stackrel{\ell}{\ell} L_{2}^{(m) *}(0,1)\right\|^{2}=(-1)^{m+1}\left[\frac{B_{2 m} h^{2 m}}{(2 m) !}-\frac{2 h^{2 m+1}}{(2 m) !} \sum_{k=1}^{m-1} d_{k} \sum_{i=0}^{2 m} \frac{q_{k}+q_{k}^{N+i}(-1)^{i+1}}{\left(q_{k}-1\right)^{i+1}} \Delta^{i} 0^{2 m}\right],
$$

where $d_{k}$ are determined from system (3.11)-(3.13), $B_{2 m}$ are Bernoulli numbers, $\Delta^{i} 0^{2 m}$ is given in Theorem 3.1, $q_{k}$ are given in Theorem 3.2.

In the proof of Theorem 3.5 we use the following relations [6]

$$
\sum_{\gamma=0}^{n-1} q^{\gamma} \gamma^{k}=\frac{1}{1-q} \sum_{i=0}^{k}\left(\frac{q}{1-q}\right)^{i} \Delta^{i} 0^{k}-\left.\frac{q^{n}}{1-q} \sum_{i=0}^{k}\left(\frac{q}{1-q}\right)^{i} \Delta^{i} \gamma^{k}\right|_{\gamma=n},
$$

where $\Delta^{i} \gamma^{k}$ is the finite difference of $\gamma^{k}$ of order $i, q$ is the ratio of a geometric progression. Let us consider the following well known relation [5]

$$
\sum_{\gamma=0}^{\beta-1} \gamma^{k}=\sum_{j=1}^{k+1} \frac{k ! B_{k+1-j}}{j !(k+1-j) !} \beta^{j}
$$

where $B_{k+1-j}$ are Bernoulli numbers

$$
\Delta^{\alpha} x^{\nu}=\sum_{p=0}^{\nu} C_{\nu}^{p} \Delta^{\alpha} 0^{p} x^{\nu-p}
$$

Proof of Theorem 3.5. Let us consider the first sum of equation (2.2). For this sum we have 


$$
\begin{aligned}
S=\sum_{\gamma=0}^{N} C[\gamma] \frac{|h \beta-h \gamma|^{2 m-1}}{2(2 m-1) !}= \\
=C[0] \frac{(h \beta)^{2 m-1}}{(2 m-1) !}+\sum_{\gamma=1}^{\beta} C[\gamma] \frac{(h \beta-h \gamma)^{2 m-1}}{(2 m-1) !}-\sum_{\gamma=0}^{N} C[\gamma] \frac{(h \beta-h \gamma)^{2 m-1}}{2(2 m-1) !} .
\end{aligned}
$$

Let us introduce

$$
S_{1}=\sum_{\gamma=1}^{\beta} C[\gamma] \frac{(h \beta-h \gamma)^{2 m-1}}{(2 m-1) !}, \quad S_{2}=\sum_{\gamma=0}^{N} C[\gamma] \frac{(h \beta-h \gamma)^{2 m-1}}{2(2 m-1) !} .
$$

Using (3.4) and (3.14), (3.15), for $S_{1}$ we have

$$
\begin{aligned}
S_{1} & =\sum_{\gamma=1}^{\beta} h\left(1+\sum_{k=1}^{m-1}\left(d_{k} q_{k}^{\gamma}+p_{k} q_{k}^{N-\gamma}\right)\right) \frac{(h \beta-h \gamma)^{2 m-1}}{(2 m-1) !}= \\
& =\frac{h^{2 m}}{(2 m-1) !}\left[\sum_{\gamma=0}^{\beta-1} \gamma^{2 m-1}+\sum_{k=1}^{m-1}\left(d_{k} q_{k}^{\beta} \sum_{\gamma=0}^{\beta-1} q_{k}^{-\gamma} \gamma^{2 m-1}+p_{k} q_{k}^{N-\beta} \sum_{\gamma=0}^{\beta-1} q_{k}^{\gamma} \gamma^{2 m-1}\right)\right]= \\
& =\frac{h^{2 m}}{(2 m-1) !}\left[\sum_{j=1}^{2 m} \frac{(2 m-1) ! B_{2 m-j}}{j ! \cdot(2 m-j) !} \beta^{j}+\sum_{k=1}^{m-1}\left[d _ { k } q _ { k } ^ { \beta } \left\{\frac{q_{k}}{q_{k}-1} \sum_{i=0}^{2 m-1} \frac{\Delta^{i} 0^{2 m-1}}{\left(q_{k}-1\right)^{i}}-\right.\right.\right. \\
& \left.-\frac{q_{k}^{1-\beta}}{q_{k}-1} \sum_{i=0}^{2 m-1} \frac{\Delta^{i} \beta^{2 m-1}}{\left(q_{k}-1\right)^{i}}\right\}+p_{k} q_{k}^{N-\beta}\left\{\frac{1}{1-q_{k}} \sum_{i=0}^{2 m-1}\left(\frac{q_{k}}{q_{k}-1}\right)^{i} \Delta^{i} 0^{2 m-1}-\right. \\
& \left.\left.-\frac{q_{k}^{\beta}}{1-q_{k}} \sum_{i=0}^{2 m-1}\left(\frac{q_{k}}{q_{k}-1}\right)^{i} \Delta^{i} \beta^{2 m-1}\right\}\right] .
\end{aligned}
$$

Taking into account that $q_{k}$ is the root of the Euler-Frobenius polynomial $E_{2 m-2}(q)$ and using Theorem 3.1, relation (3.16), the expression for $S_{1}$ is reduced to the following form

$$
\begin{aligned}
S_{1}= & \frac{(h \beta)^{2 m}}{(2 m) !}+h \cdot \frac{(h \beta)^{2 m-1}}{(2 m-1) !} B_{1}+h^{2 m} \sum_{j=1}^{2 m-2} \frac{B_{2 m-j}}{j !(2 m-j) !} \beta^{j}+ \\
& +h^{2 m} \sum_{j=0}^{2 m-1} \frac{\beta^{2 m-1-j}}{j !(2 m-1-j) !} \sum_{k=1}^{m-1} \sum_{i=0}^{j} \frac{-d_{k} q_{k}+p_{k} q_{k}^{N+i}(-1)^{i}}{\left(q_{k}-1\right)^{i+1}} \Delta^{i} 0^{j}
\end{aligned}
$$

Now we consider $S_{2}$. Using equations (2.4)-(2.7), we rewrite the expression for $S_{2}$ in powers of $h \beta$

$$
\begin{aligned}
S_{2}= & \sum_{\gamma=0}^{N} C[\gamma] \frac{(h \beta-h \gamma)^{2 m-1}}{2(2 m-1) !}= \\
= & \sum_{k=0}^{2} \sum_{j=2 k}^{2 k+1} \frac{(h \beta)^{2 m-1-j}(-1)^{j}}{2(2 m-1-j) j !}\left(\frac{1}{j+1}+\sum_{n=1}^{k} j(j-1) \ldots(j-2 n+2) A_{n}\right)+ \\
& +\sum_{j=6}^{m-1} \frac{(h \beta)^{2 m-1-j}(-1)^{j}}{2(2 m-1-j) j !}\left(\frac{1}{j+1}+\sum_{n=1}^{3} j(j-1) \ldots(j-2 n+2) A_{n}\right)+ \\
& +\sum_{j=m}^{2 m-1} \frac{(h \beta)^{2 m-1-j}(-1)^{j}}{2(2 m-1-j) ! j !} \sum_{\gamma=0}^{N} C[\gamma](h \gamma)^{j} .
\end{aligned}
$$


Let us substitute (2.8) and $S$ into equation (2.2). Then using (3.17), (3.18) and equating coefficients of the same powers of $h \beta$ we have

$$
\begin{aligned}
& \sum_{j=0}^{m-1} \lambda_{j}(h \beta)^{j}=\sum_{j=0}^{m-1} \frac{(h \beta)^{j}}{j !}\left[\frac{(-1)^{j}}{2(2 m-j) !}+\sum_{n=1}^{3} A_{n} \frac{(-1)^{j}}{2(2 m-2 n-j) !}+\right. \\
& +\frac{h^{2 m-j}}{(2 m-1-j) !} \sum_{k=1}^{m-1} \sum_{i=0}^{2 m-1-j} \frac{d_{k} q_{k}+p_{k} q_{k}^{N+i}(-1)^{i+1}}{\left(q_{k}-1\right)^{i+1}} \Delta^{i} 0^{2 m-1-j}+ \\
& \left.+\frac{(-1)^{2 m-1-j}}{2(2 m-1-j) !} \sum_{\gamma=0}^{N} C[\gamma](h \gamma)^{2 m-1-j}\right]-\sum_{j=1}^{m-1} \frac{B_{2 m-j} h^{2 m-j}(h \beta)^{j}}{j !(2 m-j) !}, \\
& \sum_{k=1}^{m-1} \sum_{i=0}^{j} \frac{d_{k} q_{k}+p_{k} q_{k}^{N+i}(-1)^{i+1}}{\left(q_{k}-1\right)^{i+1}} \Delta^{i} 0^{j}=\frac{B_{j+1}}{j+1}, \quad j=\overline{6, m-1}, \\
& \sum_{k=1}^{m-1} \sum_{i=0}^{2} \frac{d_{k} q_{k}+p_{k} q_{k}^{N+i}(-1)^{i+1}}{\left(q_{k}-1\right)^{i+1}} \Delta^{i} 0^{2}=0 \\
& \sum_{k=1}^{m-1} \sum_{i=0}^{4} \frac{d_{k} q_{k}+p_{k} q_{k}^{N+i}(-1)^{i+1}}{\left(q_{k}-1\right)^{i+1}} \Delta^{i} 0^{4}=0, \\
& C[0]=h\left(\frac{1}{2}+\sum_{k=1}^{m-1} \frac{p_{k} q_{k}^{N}-d_{k} q_{k}}{1-q_{k}}\right), \\
& A_{n}=\frac{h^{2 n} B_{2 n}}{(2 n) !}-\frac{h^{2 n}}{(2 n-1) !} \sum_{k=1}^{m-1} \sum_{i=1}^{2 n-1} \frac{d_{k} q_{k}+p_{k} q_{k}^{N+i}(-1)^{i+1}}{\left(q_{k}-1\right)^{i+1}} \Delta^{i} 0^{2 n-1}, n=1,2,3 .
\end{aligned}
$$

Taking into account (3.23) and (3.4), when $\alpha=0$ we obtain from (2.4)

$$
C[N]=h\left(\frac{1}{2}+\sum_{k=1}^{m-1} \frac{d_{k} q_{k}^{N}-p_{k} q_{k}}{1-q_{k}}\right) .
$$

Substituting $\lambda_{j}$ from (3.19) into (2.3), we obtain the following equations for unknowns $d_{k}$ and $p_{k}$

$$
\begin{aligned}
\sum_{k=1}^{m-1} \sum_{i=0}^{2 m-2} & \frac{d_{k} q_{k}^{i}+p_{k} q_{k}^{N+1}(-1)^{i+1}}{\left(1-q_{k}\right)^{i+1}} \Delta^{i} 0^{2 m-2}=\sum_{k=1}^{m-1} \sum_{i=0}^{2 m-2} \frac{d_{k} q_{k}^{N+i}+p_{k} q_{k}(-1)^{i+1}}{\left(1-q_{k}\right)^{i+1}} \Delta^{i} 0^{2 m-2} \\
& \sum_{k=1}^{m-1} \sum_{i=0}^{j-1} \frac{d_{k} q_{k}^{N+i}+p_{k} q_{k}(-1)^{i+1}}{\left(1-q_{k}\right)^{i+1}} \Delta^{i} 0^{j-1}=\frac{B_{j}}{j}, j=\overline{7, m} \\
& \sum_{k=1}^{m-1} \sum_{i=0}^{3} \frac{d_{k} q_{k}+p_{k} q_{k}^{N+i}(-1)^{i+1}}{\left(q_{k}-1\right)^{i+1}} \Delta^{i} 0^{3}=\sum_{k=1}^{m-1} \sum_{i=0}^{3} \frac{d_{k} q_{k}^{N+i}+p_{k} q_{k}(-1)^{i+1}}{\left(1-q_{k}\right)^{i+1}} \Delta^{i} 0^{3} \\
& \sum_{k=1}^{m-1} \sum_{i=0}^{5} \frac{d_{k} q_{k}+p_{k} q_{k}^{N+i}(-1)^{i+1}}{\left(q_{k}-1\right)^{i+1}} \Delta^{i} 0^{5}=\sum_{k=1}^{m-1} \sum_{i=0}^{5} \frac{d_{k} q_{k}^{N+i}+p_{k} q_{k}(-1)^{i+1}}{\left(1-q_{k}\right)^{i+1}} \Delta^{i} 0^{5} \\
& \sum_{k=1}^{m-1} \sum_{i=0}^{2} \frac{d_{k} q_{k}^{N+i}+p_{k} q_{k}(-1)^{i+1}}{\left(1-q_{k}\right)^{i+1}} \Delta^{i} 0^{2}=0
\end{aligned}
$$




$$
\begin{gathered}
\sum_{k=1}^{m-1} \sum_{i=0}^{4} \frac{d_{k} q_{k}^{N+i}+p_{k} q_{k}(-1)^{i+1}}{\left(1-q_{k}\right)^{i+1}} \Delta^{i} 0^{4}=0, \\
\sum_{k=1}^{m-1} \frac{d_{k} q_{k}+p_{k} q_{k}^{N+1}}{\left(q_{k}-1\right)^{2}}=\sum_{k=1}^{m-1} \frac{d_{k} q_{k}^{N+1}+p_{k} q_{k}}{\left(1-q_{k}\right)^{2}}, \\
\sum_{k=1}^{m-1} \sum_{i=0}^{2 m-4} \frac{d_{k} q_{k}^{i}+p_{k} q_{k}^{N+1}(-1)^{i+1}}{\left(1-q_{k}\right)^{i+1}} \Delta^{i} 0^{2 m-4}=\sum_{k=1}^{m-1} \sum_{i=0}^{2 m-4} \frac{d_{k} q_{k}^{N+i}+p_{k} q_{k}(-1)^{i+1}}{\left(1-q_{k}\right)^{i+1}} \Delta^{i} 0^{2 m-4}, \\
\sum_{k=1}^{m-1} \sum_{i=0}^{2 m-6} \frac{d_{k} q_{k}^{i}+p_{k} q_{k}^{N+1}(-1)^{i+1}}{\left(1-q_{k}\right)^{i+1}} \Delta^{i} 0^{2 m-6}=\sum_{k=1}^{m-1} \sum_{i=0}^{2 m-6} \frac{d_{k} q_{k}^{N+i}+p_{k} q_{k}(-1)^{i+1}}{\left(1-q_{k}\right)^{i+1}} \Delta^{i} 0^{2 m-6} .
\end{gathered}
$$

Thus, from (3.20)-(3.21), (3.22) and (3.26)-(3.34) we obtain

$$
\begin{aligned}
& \sum_{k=1}^{m-1}\left(d_{k}-p_{k}\right) \sum_{i=0}^{2 m-2 j} \frac{q_{k}+q_{k}^{N+i}(-1)^{i+1}}{\left(q_{k}-1\right)^{i+1}} \Delta^{i} 0^{2 m-2 j}=0, \quad j=1,2,3, \\
& \sum_{k=1}^{m-1}\left(d_{k}-p_{k}\right) \sum_{i=0}^{2 j} \frac{q_{k}+q_{k}^{N+i}(-1)^{i+1}}{\left(q_{k}-1\right)^{i+1}} \Delta^{i} 0^{2 j}=0, \quad j=1,2, \\
& \sum_{k=1}^{m-1}\left(d_{k}-p_{k}\right) \sum_{i=0}^{j} \frac{q_{k}+q_{k}^{N+i}(-1)^{i+1}}{\left(q_{k}-1\right)^{i+1}} \Delta^{i} 0^{j}=0, \quad j=\overline{6, m-1} .
\end{aligned}
$$

Taking into account uniqueness of the optimal coefficients, we conclude that homogeneous system of linear equations (3.35)-(3.37) has trivial solution. This means that

$$
d_{k}=p_{k}, \quad k=1,2, \ldots, m-1 .
$$

Then using (3.38), from (3.35)-(3.37) we obtain (3.11)-(3.13), and from (3.4), (3.23)-(3.24), (3.25) we obtain (3.7)-(3.10).

Theorem 3.5 is proved.

Remark 1. It should be noted that in the cases $m=6$ and $m=7$ we obtain from Theorems 3.5 and 3.5 the classical Euler-Maclaurin quadrature formula.

\section{References}

[1] P.Blaga, Gh.Coman, Some problems on optimal quadrature, Stud. Univ. Babeş-Bolyai Math., 52(2007), no. 4, 21-44.

[2] B.Bojanov, Optimal quadrature formulas, Russian Mathematical Surveys, 60(2005), no. 6, $1035-1055$.

[3] T.Catinaş, Gh. Coman, Optimal quadrature formulas based on the $\phi$-function method, Stud. Univ. Babeş-Bolyai Math., 51(2006), no. 1, 49-64.

[4] M.A.Chakhkiev, Linear differential operators with real spectrum, and optimal quadrature formulas, Izv. Akad. Nauk SSSR, Ser. Mat., 48(1984), no. 5, 1078-1108 (in Russian).

[5] A.O.Gelfond, Calculus of Finite Differences, Nauka, Moscow, 1967 (in Russian).

[6] R.W.Hamming, Numerical methods for Scientists and Engineers, McGraw Bill Book Company, Inc., USA, 1962. 
[7] A.R.Hayotov, G.V.Milovanović, Kh.M.Shadimetov, On an optimal quadrature formula in the sense of Sard, Numerical Algorithms, 57(2011), no. 4, 487-510.

[8] P.Köhler, On the weights of Sard's quadrature formulas, Calcolo, 25(1988), 169-186.

[9] F.Lanzara, On optimal quadrature formulae, J. Ineq. Appl., 5(2000), 201-225.

[10] L.F.Meyers, A.Sard, Best approximate integration formulas, J. Math. Physics, 29(1950), 118-123.

[11] S.M.Nikol'skii, To question about estimation of approximation by quadrature formulas, Uspekhi Matem. Nauk, 36(1950), no. 5:2, 165-177 (in Russian).

[12] S.M.Nikol'skii, Quadrature Formulas, Nauka, Moscow, 1988 (in Russian).

[13] A.Sard, Best approximate integration formulas; best approximation formulas, Amer. J. Math., 71(1949), 80-91.

[14] A.Sard, Linear approximation, AMS, 1963.

[15] I.J.Schoenberg, On monosplines of least deviation and best quadrature formulae, J. Soc. Indust. Appl. Math. Ser. B Numer. Anal., 2(1965), 144-170.

[16] I.J.Schoenberg, S.D.Silliman, On semicardinal quadrature formulae, Math. Comp., 126(1974), 483-497.

[17] Kh.M.Shadimetov, Optimal formulas of approximate integration for differentiable functions, Candidate dissertation, Novosibirsk, 1983, arXiv:1005.0163v1 [NA.math].

[18] Kh.M.Shadimetov, Optimal quadrature formulas in $L_{2}^{m}(\Omega)$ and $L_{2}^{m}\left(R^{1}\right)$, Dokl. Akad. Nauk UzSSR, (1983), no. 3, 5-8 (in Russian).

[19] Kh.M.Shadimetov. The discrete analogue of the differential operator $d^{2 m} / d x^{2 m}$ and its construction, Questions of Computations and Applied Mathematics. Tashkent, 1985, 22-35. ArXiv:1001.0556.v1 [math.NA] Jan. 2010.

[20] Kh.M.Shadimetov, Construction of weight optimal quadrature formulas in the space $L_{2}^{(m)}(0, N)$, Siberian J. Comput. Math., 5(2002), no. 3, 275-293 (in Russian).

[21] Kh.M.Shadimetov, A.R.Hayotov, Optimal quadrature formulas with positive coefficients in $L_{2}^{(m)}(0,1)$ space, J. Comput. Appl. Math., 235(2011), 1114-1128.

[22] Kh.M.Shadimetov, A.R.Hayotov, Optimal quadrature formulas in the sense of Sard in $W_{2}^{(m, m-1)}$ space, Calcolo, 51(2014), 211-243.

[23] Kh.M.Shadimetov, A.R.Hayotov, F.A.Nuraliev, On an optimal quadrature formula in Sobolev space $L_{2}^{(m)}(0,1)$, J. Comput. Appl. Math., 243(2013), 91-112.

[24] S.L.Sobolev, Introduction to the Theory of Cubature Formulas, Nauka, Moscow, 1974 (in Russian).

[25] S.L.Sobolev, The coefficients of optimal quadrature formulas, Selected Works of S. L. Sobolev, Springer, 2006, 561-566.

[26] S.L.Sobolev, V.L.Vaskevich, The Theory of Cubature Formulas, Kluwer Academic Publishers Group, Dordrecht, 1997. 
[27] F.Ya.Zagirova, On construction of optimal quadrature formulas with equal spaced nodes, Novosibirsk, 1982, (Preprint no. 25, Institut Mat., Sib. Otd. AN SSSR) (in Russian).

[28] Z.Zh.Zhamalov, Kh.M.Shadimetov, About optimal quadrature formulas, Dokl. Akademii Nauk UzSSR, 7(1980), 3-5 (in Russian).

[29] A.A.Zhensikbaev, Monosplines of minimal norm and the best quadrature formulas, Uspekhi Matem. Nauk, 36(1981), 107-159 (in Russian).

\title{
Оптимальные формулы численного интегрирования с производными в пространстве Соболева
}

\author{
Холмат М. Шадиметов \\ Фарход А. Нуралиев \\ Институт математики имени В. И. Романовского \\ Академии наук Республики Узбекистан \\ Мирзо Улугбека, 81, Ташкент, 100041 \\ Узбекистан
}

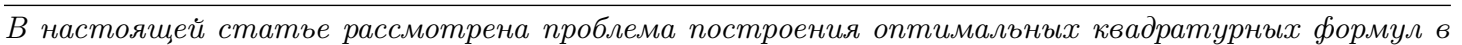
смысле Сарда в пространстве $L_{2}^{(m)}(0,1)$. Здесъ квадратурная сумма состоит из значений подынтегральной функции в узловых точках и значений первой, третъей и пятой производных подынтегральной функиии в кониевых точках интервала инегрирования. Найдены коэфбициенты оптимальных квадратурных формул и вычислена норма оптимального функционала погрешности для любого натурального $N$ и для любого $m \geqslant 6$ с использованием метода Соболева, основанном на дискретном аналоге дифференциального оператора $d^{2 m} / d x^{2 m}$. В частности, для $m=6,7$ оптимальность классической квадратурной формуль Эйлера-Маклорена доказана. Начиная с $m=8$ получены новые оптималъные квадратурные формулы.

Ключевые слова: оптимальная квадратурная формула, функционал погрешности, экстремальная функиия, оптимальные коэффициенты. 